Fluorescent biopsy of biological tissues in differentiation of benign and malignant tumors of prostate

L. I. Trifoniuk

Yu. A. Ushenko

M. I. Sidor

O. P. Minzer

M. V. Gritsyuk

O. Y. Novakovskaya 


\title{
Fluorescent biopsy of biological tissues in differentiation of benign and malignant tumors of prostate
}

\author{
Trifoniuk L.I. ${ }^{1}$, Ushenko Yu.O. ${ }^{2}$, Sidor M.I. ${ }^{3}$, Minzer O.P. ${ }^{1}$, \\ Gritsiuk M.V. ${ }^{1}$, Novakovskaya O.Y. ${ }^{1}$ \\ ${ }^{1}$ Bukovinian State Medical University, Chernivtsi, 58000, Ukraine \\ ${ }^{2}$ Correlation Optics Department, Chernivtsi National University, \\ 2 Kotsyubinsky Str., Chernivtsi, 58012, Ukraine \\ ${ }^{3}$ Optics and Publishing Department, Chernivtsi National University, \\ 2 Kotsyubinsky Str., Chernivtsi, 58012, Ukraine \\ a.dubolazov@,chnu.edu.ua
}

\begin{abstract}
The work consists of investigation results of diagnostic efficiency of a new azimuthally stable Mueller-matrix method of analysis of laser autofluorescence coordinate distributions of biological tissues histological sections. A new model of generalized optical anisotropy of biological tissues protein networks is proposed in order to define the processes of laser autofluorescence. The influence of complex mechanisms of both phase anisotropy (linear birefringence and optical activity) and linear (circular) dichroism is taken into account. The interconnections between the azimuthally stable Mueller-matrix elements characterizing laser autofluorescence and different mechanisms of optical anisotropy are determined. The statistic analysis of coordinate distributions of such Mueller-matrix rotation invariants is proposed. Thereupon the quantitative criteria (statistic moments of the 1st to the 4th order) of differentiation of histological sections of uterus wall tumor - group 1 (dysplasia) and group 2 (adenocarcinoma) are estimated.
\end{abstract}

Keywords: fluorescent biopsy, biological tissues, malignant tumors of prostate.

\section{INTRODUCTION}

Biological tissues represent structurally heterogeneous optical anisotropic media with absorption. To describe interactions of polarized light with such complex systems more generalized approximations are required based on Mueller-matrix formalism. Nowadays many practical techniques based on the measurement and analyses of Mueller matrices of the investigated samples are applied in biological and medical research. [1-5]. A separate direction - laser polarimetry - was formed in matrix optics within 10-15 years [6-10].

In parallel to polarimetric the fluorescence methods are actively being developed. Such methods are grounded on the diagnostic utilization of fluorescence effects of protein molecules and their complexes. Consequently, the valid results concerning the cancer fluorescence diagnostic of cavitary organs were obtained [11-16].

In this research the model of generalized optical anisotropy inherent for the tissues of women's reproductive sphere is suggested and on its basis the method of Mueller-matrix mapping of laser polarization autofluorescence of histological sections of biopsy of benign (dysplasia) and malignant (adenocarcinoma) tumors of uterus wall is applied.

Biosensing and Nanomedicine VII, edited by Hooman Mohseni, Massoud H. Agahi, Manijeh Razeghi, Proc. of SPIE Vol. 9166, 916618 - (C) 2014 SPIE - CCC code: 0277-786X/14/\$18 - doi: 10.1117/12.2061277 


\section{BRIEF THEORY}

In this work we have limit oneself to consider spectral-selective case - luminiscence of optically active porphyrins of biological tissue in red $\left(\lambda_{f}=0.63-0.65 \mu \mathrm{m}\right)$ region of spectrum [17-31]. The excitation of autofluorescence was realized by blue solid-state laser with the wavelength $\lambda=0.405 \mu \mathrm{m}$, which coincide with the porphyrins maximum absorption.

The following model ideas concerning optical anisotropy of protein networks are used as the basis for the description of laser polarization autofluorescence of biological tissues.

The formation of laser polarization autofluorescence is based on the mechanisms of optically anisotropic absorption (linear and circular dichroism) [18]; fluorescence of porphyrin molecules ("linear" oscillators) and generated by them networks ("elliptical" oscillators) [19] and mechanisms of phase anisotropy (linear and circular birefringence) that modulate the fluorescent radiation of protein molecules and their structures. The mentioned scenario can be described by using Mueller-matrix formalism.

1. Absorption - Amino acids and polypeptide chains (primary structure of protein) made by them form the fibrillar (secondary structure) protein networks possessing the linear dichroism. Optical manifestations of such a mechanism are exhaustively described by the next Mueller matrices:

$$
\{\Psi\}=\left\|\begin{array}{cccc}
1 & \varphi_{12} & \varphi_{13} & 0 \\
\varphi_{21} & \varphi_{22} & \varphi_{23} & 0 \\
\varphi_{31} & \varphi_{32} & \varphi_{33} & 0 \\
0 & 0 & 0 & \varphi_{44}
\end{array}\right\|, \text { where } \varphi_{i k}=\left\{\begin{array}{l}
\varphi_{12}=\varphi_{21}=(1-\Delta \tau) \cos 2 \rho \\
\varphi_{13}=\varphi_{31}=(1-\Delta \tau) \sin 2 \rho \\
\varphi_{22}=(1+\Delta \tau) \cos ^{2} 2 \rho+2 \sqrt{\Delta \tau} \sin ^{2} 2 \rho \\
\varphi_{23}=\varphi_{32}=(1-\Delta \tau) \sin 2 \rho \\
\varphi_{33}=(1+\Delta \tau) \sin ^{2} 2 \rho+2 \sqrt{\Delta \tau} \cos ^{2} 2 \rho \\
\varphi_{44}=2 \sqrt{\Delta \tau}
\end{array}\right.
$$

Here $\Delta \tau=\frac{\tau_{x}}{\tau_{y}},\left\{\begin{array}{l}\tau_{x}=\tau \cos \rho ; \\ \tau_{y}=\tau \sin \rho\end{array}, \tau_{x}, \quad \tau_{y}\right.$ - absorption coefficients of linearly polarized orthogonal components of the light beam amplitude.

Availability of complex spiral-like structures or their combinations (third-order structure) of polypeptide protein structures forms the circular dichroism. Optical manifestations of such configuration peculiarities are characterized by the Mueller matrix:

$$
\{\Phi\}=\left\|\begin{array}{cccc}
1 & 0 & 0 & \phi_{14} \\
0 & \phi_{22} & 0 & 0 \\
0 & 0 & \phi_{33} & 0 \\
\phi_{41} & 0 & 0 & 1
\end{array}\right\|, \text { where } \phi_{i k}=\left\{\begin{array}{l}
\phi_{22}=\phi_{33}=\frac{1-\Delta g^{2}}{1+\Delta g^{2}} \\
\phi_{14}=\phi_{41}= \pm \frac{2 \Delta g}{1+\Delta g^{2}}
\end{array}\right.
$$

Here $\Delta g=\frac{g_{\otimes}-g_{\oplus}}{g_{\otimes}+g_{\oplus}}, g_{\otimes}, \quad g_{\oplus}-$ absorption indices of left- $(\otimes)$ and right-hand $(\oplus)$ circularly polarized components of light beam amplitude. 
2. Fluorescence - Polarization appearance of porphyrin fluorescence is characterized by Mueller-matrix provided in $[20]$

$$
\{F\}=\left\|\begin{array}{cccc}
1 & F_{12} & 0 & 0 \\
F_{21} & F_{22} & 0 & 0 \\
0 & 0 & F_{33} & 0 \\
0 & 0 & 0 & F_{44}
\end{array}\right\| \text {, where } F_{i k}=F_{11}^{-1}\left\{\begin{array}{l}
F_{11}=a-b \sin ^{2} \vartheta ; \\
F_{12}=F_{21}=-b \sin ^{2} \vartheta ; \\
F_{22}=b\left(1+\cos ^{2} \vartheta\right) ; \\
F_{33}=2 b \cos \vartheta ; \\
F_{44}=2 c \cos \vartheta .
\end{array} .\right.
$$

Here $\vartheta$ - is a scattering angle; $a$ and $b$ - are the interconnected constants for the system of linear oscillators in isotropic medium, defined by following relations

$$
\begin{aligned}
& a=0.5\left(1+\left\langle\cos ^{2} \varepsilon\right\rangle\right), \\
& b=0.25\left(3\left\langle\cos ^{2} \varepsilon\right\rangle-1\right),
\end{aligned}
$$

where $\varepsilon$ is the angle between the emission dipole and the azimuth of the polarization of the exciting beam. There are two experimentally important cases where the theoretical limits of $\left\langle\cos ^{2} \varepsilon\right\rangle$ can be predicted [20]: if the emission and absorption dipoles are collinear $\left\langle\cos ^{2} \varepsilon\right\rangle=3 / 5$, and if the emission dipoles are randomly aligned $\left\langle\cos ^{2} \varepsilon\right\rangle=1 / 3$.

The parameter $c$ is undoubtedly related to optical activity. From a classical viewpoint a "circular oscillator" would be caused by the induced electric and magnetic transition dipole moments being fully parallel or antiparallel and having the same magnitude. Following this description, optically active liquid-crystal molecules are "elliptical oscillators". The limiting values of $c$ for such a system would be $c=5 / 16$.

3. Phase modulation of fluorescence. Amino acids and polypeptide chains made by them (primary structure of protein) demonstrate optical activity and are characterized by the following matrix operator

$$
\{\Omega\}=\left\|\begin{array}{cccc}
1 & 0 & 0 & 0 \\
0 & \omega_{22} & \omega_{23} & 0 \\
0 & \omega_{32} & \omega_{33} & 0 \\
0 & 0 & 0 & 1
\end{array}\right\| \text {, where } \omega_{i k}=\left\{\begin{array}{l}
\omega_{22}=\omega_{33}=\cos 2 \gamma \\
\omega_{23}=-\omega_{32}=\sin 2 \gamma
\end{array}\right.
$$

Here $\gamma$ - rotation angle of polarization plane fluorescent radiation.

Fibrillar (secondary structure) protein networks formed by polypeptide chains possess linear birefringence and are exhaustively described by the Mueller matrix

$$
\{D\}=\left\|\begin{array}{cccc}
1 & 0 & 0 & 0 \\
0 & d_{22} & d_{23} & d_{24} \\
0 & d_{32} & d_{33} & d_{34} \\
0 & d_{42} & d_{43} & d_{44}
\end{array}\right\|, \text { where } d_{i k}=\left\{\begin{array}{l}
d_{22}=\cos ^{2} 2 \rho+\sin ^{2} 2 \rho \cos \delta ; \\
d_{23}=d_{32}=\cos 2 \rho \sin 2 \rho(1-\cos \delta) ; \\
d_{33}=\sin ^{2} 2 \rho+\cos ^{2} 2 \rho \cos \delta ; \\
d_{24}=-d_{42}=\sin 2 \rho \sin \delta ; \\
d_{34}=-d_{43}=\cos 2 \rho \sin \delta ; \\
d_{44}=\cos \delta .
\end{array}\right.
$$


Here $\rho$-direction of fibril's packing, $\delta$-phase shift between linearly-polarized orthogonal components of fluorescent light amplitude.

Considering the effect of all mechanisms of optically anisotropic absorption and phase anisotropy, the Mueller matrix of laser polarization autofluorescence of the protein network of biological tissue can be written as follows

$$
\{M\}=\{D\} \Omega\}\{\{F\} \Psi \Psi\}\{\Phi\}=\left\|\begin{array}{cccc}
1 & M_{12} & M_{13} & M_{14} \\
M_{21} & M_{22} & M_{23} & M_{24} \\
M_{31} & M_{32} & M_{33} & M_{34} \\
M_{41} & M_{42} & M_{43} & M_{44}
\end{array}\right\| .
$$

The analysis of matrix (8) shows that elements $M_{i k}$ characterize the superposition of mechanisms of linear $(\Delta \tau)$ and circular $(\Delta g)$ dichroism; fluorescence of linear $\left(F_{12 ; 21 ; 22 ; 33}\right)$ and elliptical $\left(F_{44}\right)$ oscillators with the following phase modulation of such a radiation by optically active molecules $(\theta)$ and birefringent $(\delta)$ networks of them.

The "information content" of matrix elements is different. The set of elements $M_{i=1 ; k=1 ; 2 ; 3 ; 4}\left(F_{12}\right)$ characterizes the fluorescence of linear oscillators originated due to the anisotropic absorption. The elements $M_{i=2 ; 3 ; k=1 ; 2 ; 3 ;}\left(F_{21 ; 22 ; 33}\right)$ define the phase-modulated $(\delta, \theta)$ fluorescence of linear oscillators. Finally, the values of elements $M_{i=4 ; k=1 ; 2 ; 3 ; 4}\left(F_{21 ; 22 ; 33}, F_{44}\right)$ consist of complex information concerning the fluorescence of linear $\left(F_{21 ; 22 ; 33}\right)$ and elliptical $\left(F_{44}\right)$ oscillators in optically anisotropic medium with linear and circular birefringence.

It should be noted that not all elements of matrix (8) suitable for practical usage. The reason for that consists in azimuthally dependence of most matrix elements - in general, 12 of 16 elements change at rotation of the sample around the probing axis. It is shown in $[2,4]$ that the following elements of the matrix $\{M\}$, as well as their combinations, are azimuthally stable, independent of the rotation angle $(\Theta)$ of the sample

$$
\left\{\begin{array}{l}
M_{11}(\Theta)=\text { const } \\
M_{14}(\Theta)=\text { const } \\
M_{41}(\Theta)=\text { const } \\
M_{44}(\Theta)=\text { const }
\end{array}\right.
$$

The analysis of the above presented rotation invariants (9) shows, that by measuring of $\left\{M_{14}=W\left(F_{12}(a, b) ; \varphi_{i k}(\Delta \tau) ; \phi_{i k}(\Delta g)\right)\right.$ $\left\{M_{41}=Q\left(F_{21 ; 22 ; 33}(a, b) ; F_{44}(c) ; \varphi_{i k}(\Delta \tau) ; \phi_{i k}(\Delta g) ; d_{i k}(\delta) ; \omega_{i k}(\theta)\right)\right.$ it is possible to obtain separated and azimuthally-stable information about laser polarization fluorescence of linear $\left(F_{12 ; 21 ; 22 ; 33}\right)$ and elliptical $\left(F_{44}\right)$ oscillators, excited by mechanisms of linear and circular dichroism of laser radiation in optically anisotropic biological tissue.

\section{ANALYSIS AND DISCUSSION OF EXPERIMENTAL RESULTS}

Optically thin (the geometric thickness $d=30 \mu \mathrm{m}$, attenuation coefficient $\tau<0.1$ ) histological sections of postsurgical biopsy of uterus wall tumors of two types were used as objects of investigation:

- $\quad$ benign tumor (dysplasia) - group 1 (21 samples);

- malignant tumor (adenocarcinoma) - group 2 (21 samples).

Histological sections were prepared according to standard technique on the freezing microtome.

The measurements of coordinate distributions of Mueller-matrix elements characterizing polarization properties of histological sections of uterus wall tumors were performed in the setup of the standard Stokes-polarimeter. The detailed description of the optical scheme and basic parts of experimental setup was presented in a series of articles [21- 
28]. In this research for autofluorescence excitation we used a diode laser with the wavelength $\lambda=0.405 \mu m$ and power $W=50 \mu \mathrm{Bm}$. For spectral division of polarization fluorescence in front of CCD-camera an interference filter was placed with the maximal transmission bandpass $\lambda_{f}=0.63-0.65 \mu \mathrm{m}$, which corresponded to intensity of laser polarization autofluorescence maximally achieved under these conditions.

The values of Mueller-matrix rotation invariants (relations (10)-(11)) were determined by means of the algorithm

$$
\left\{\begin{array}{l}
M_{14}=S_{1}^{\otimes}-0,5\left(S_{1}^{0}+S_{1}^{90}\right) \\
M_{41}=0,5\left(S_{4}^{0}+S_{4}^{90}\right)
\end{array}\right.
$$

Here $S_{i=1 ; 4}^{0 ; 90 ; \otimes}$ - Stokes vector parameters in the points of digital image of laser polarization autofluorescence of histological sections measured for a series of linearly $\left(0^{0} ; \quad 90^{\circ}\right)$ and right-hand circularly polarized $(\otimes)$ probing laser beams within the limits of 2D $(m \times n)$ ensemble of light-sensitive pixels.

For objective assessment of coordinate distributions of Mueller-matrix rotation invariants $q \equiv\left\{M_{14 ; 41}(m \times n)\right\}$ we used the traditional method of statistic analysis. The set of statistic moments of the $1^{\text {st }}-4^{\text {th }}$ orders which characterize distributions $q$ was calculated using the following algorithms

$$
Z_{1}=\frac{1}{N} \sum_{j=1}^{N}|q| ; Z_{2}=\sqrt{\frac{1}{N} \sum_{j=1}^{N}(q)_{j}{ }^{2}} ; Z_{3}=\frac{1}{Z_{2}{ }^{3}} \frac{1}{N} \sum_{j=1}^{N}(q)_{j}^{3} ; Z_{4}=\frac{1}{Z_{2}{ }^{4}} \frac{1}{N} \sum_{j=1}^{N}(q)_{j}{ }^{4} .
$$

where $N$ - number of pixels of CCD-camera.

Figures 1 and 2 present the series of experimentally measured (relations (10)) spectral-selective $\left(\Delta \lambda_{f}\right)$ Muellermatrix fluorescent images $M_{14 ; 41}(m \times n)$ characterizing laser polarization fluorescence of optically anisotropic histological sections of uterus wall tumor of group 1 (Fig. 1) and group 2 (Fig. 2).

$\mathrm{M}_{11}$

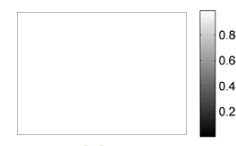

$M_{21}$

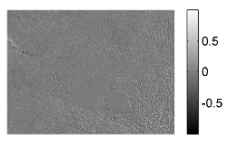

$M_{31}$

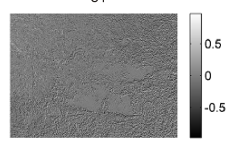

$\mathrm{M}_{41}$

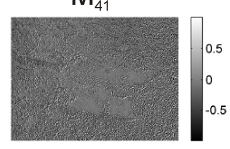

$\mathrm{M}_{12}$

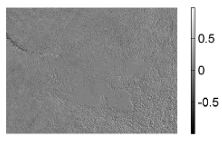

$\mathrm{M}_{22}$

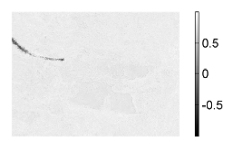

$M_{32}$

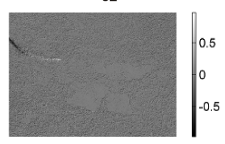

$\mathrm{M}_{42}$

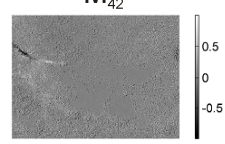

$\mathrm{M}_{13}$

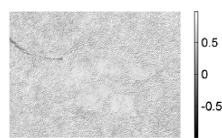

$\mathrm{M}_{23}$

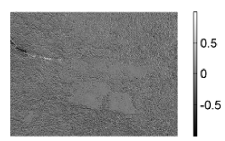

$M_{33}$

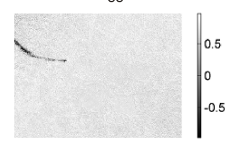

$\mathrm{M}_{43}$

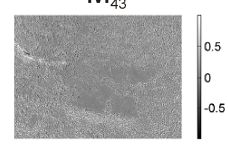

$M_{14}$

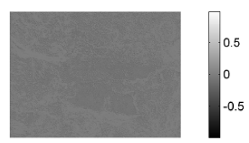

$\mathrm{M}_{21}$

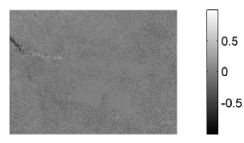

$\mathrm{M}_{34}$

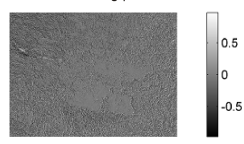

$\mathrm{M}_{44}$

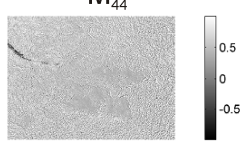

Fig. 1. Mueller-matrix fluorescent images of histological section of benign (dysplasia) tumor of uterus wall (group 1) 


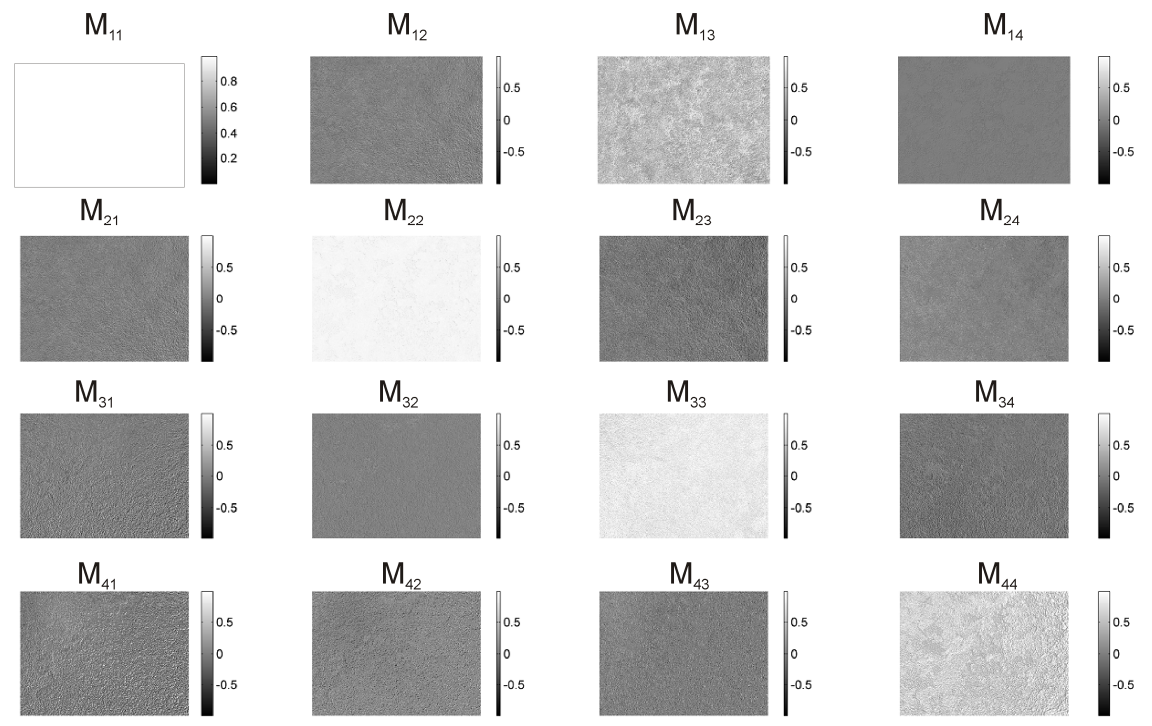

Fig. 2. Mueller-matrix fluorescent images of histological section of malignant (adenocarcinoma) tumor of uterus wall (group 2)

The analysis of the data obtained shows the common regularity - the non-zero value of all the Mueller matrices elements characterizing polarization fluorescence of histological sections of uterus wall tumor. Such a fact is experimentally confirms of model structure of Mueller-matrix (8) as superposition of matrix operators, which characterize linear and circular dichroism $(1,2)$, fluorescence of porphyrins $((3)-(5))$ and phase modulation of such a radiation $(6,7)$. However, like it was assumed during the model analysis, polarization autofluorescence is the most vividly manifested in coordinate distributions of Mueller-matrix rotation invariants $M_{41}$ and $M_{14}$ of the samples of group 1 and group 2. Such distributions $(100$ pix $\times 100$ pix $)$ are illustrated by the series of coordinate dependencies presented in Fig. 3 and Fig. 4.

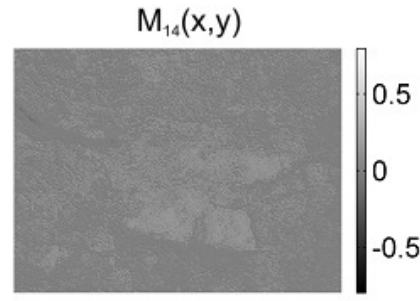

(1)

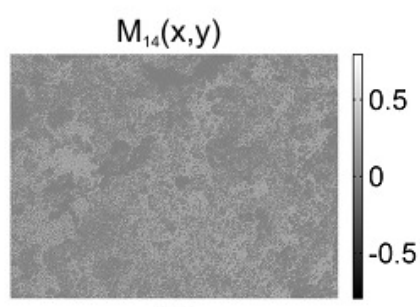

(2)
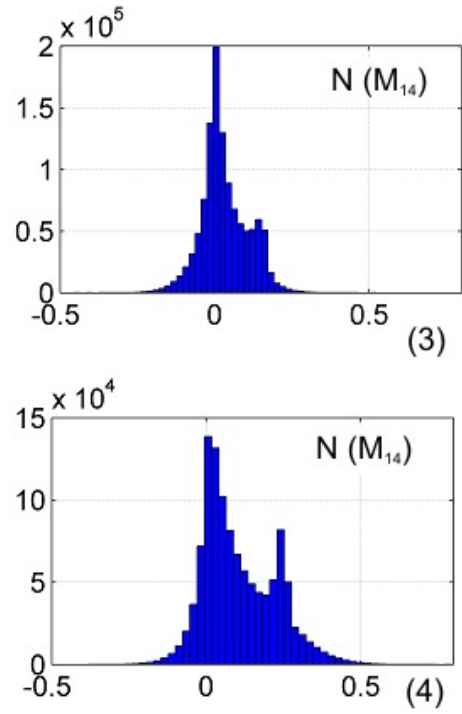
Fig. 3. Coordinate distributions of Mueller-matrix rotation invariant $M_{14}$ of optically thin histological sections of postsurgical biopsy of benign (fragments (1), (3)) and malignant (fragments (2), (4)) tumor of uterus wall

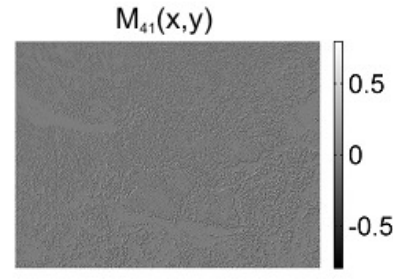

(1)

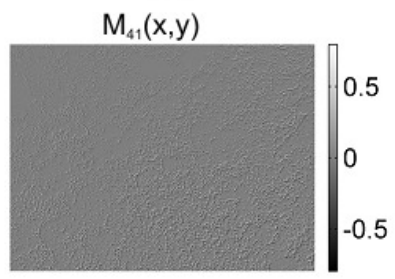

(2)

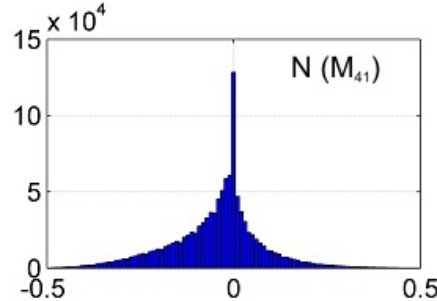

(3)

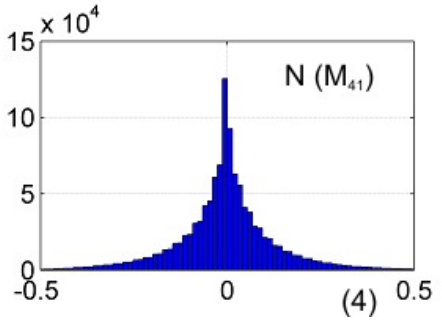

Fig. 4. Coordinate distributions of Mueller-matrix rotation invariant $M_{41}$ of optically thin histological sections of postsurgical biopsy of benign (fragments (1),(3)) and malignant (fragments (2),(4)) tumor of uterus wall

The analysis of coordinate distributions of invariant $M_{14}$ (Fig. 3), which characterizes the processes of transformation of circularly polarized fluorescent radiation into a linearly one, reveals decreasing in more than 2 times of the value $\left(M_{14} \downarrow\right)$ and the range of changes $\left(\Delta M_{14} \downarrow\right)$ of such a parameter in the plane of tissue histological section with adenocarcinoma (parts 3 and 4 ).

The inverse situation occurs for Mueller-matrix invariant $M_{41}$ (Fig. 4), which characterizes the processes of transformation of linearly polarized fluorescent radiation into a circular one.

Let us analyze the obtained results from the physical point of view. The samples of both types within the framework of considered model ((1)-(8)) represent the systems of "linear" $\left(F_{12 ; 21 ; 22 ; 33}(a, b)\right)$ and "elliptic" $\left(F_{44}(c)\right)$ fluorescent oscillators in the optically-anisotropic matrix with linear (6) and circular (7) birefringence.

As it is well known [21-31] that orientationally $(\rho)$ - phase $(\delta, \theta)$ structure of such a matrix depends on physiologically (pathological) state of biological tissue. More disordered by directions $(\Delta \rho \uparrow)$ birefringent $(\Delta n \approx$ const $)$ fibrillar network is typical for malignant states. On the other hand, in [29-31] it was shown that autofluorescence in the red region of spectrum being increased together with growth and development of tumor. This phenomenon can be connected with the liquid-crystal networks of porphyrins, which accumulate in tissues of malignant new formations on different stages of their development.

Thus, the malignant states are accompanied by formation of prevailed, over the disordered "linear" oscillators, system of fluorescent "elliptical" oscillators. Vice-versa, in the case of pre-cancer state the prevailing of "linear" fluorescent oscillators is more typical. In other words, for tissue with dysplasia the following analytical scenario is realized: $\left\{\begin{array}{l}a \uparrow, b \uparrow \rightarrow F_{12 ; 21 ; 2 ; 33}(a, b) \uparrow \Rightarrow M_{14} \Uparrow ; \\ c \downarrow \rightarrow F_{44}(c) \downarrow \Rightarrow M_{41} \Downarrow\end{array}\right.$. The inverse regularity takes place for malignant new formations: $\left\{\begin{array}{l}a \downarrow, b \downarrow \rightarrow F_{12 ; 21 ; 2 ; 33}(a, b) \downarrow \Rightarrow M_{14} \downarrow ; \\ c \uparrow \rightarrow F_{44}(c) \uparrow \Rightarrow M_{41} \Uparrow\end{array}\right.$ 
The secondary phase modulation of fluorescent radiation by fibrillar networks of both types samples does not introduce any sufficient changes in the mentioned scenario. As far as for pre-cancer and cancer states it is typical practically the same birefringence ( $\Delta n \approx$ const ) of protein structures.

The results of the quantitative statistical (relations (11)) analysis of the series of Mueller-matrix rotation invariants of both groups of histological sections are illustrated by the data presented in Table 1 .

Table 1. Statistical ( $Z_{i=1 ; 2 ; 3 ; 4}$ ) moments of the $1^{\text {st }}-4^{\text {th }}$ order distribution of Mueller-matrix invariants of histological sections of uterus wall tumor

\begin{tabular}{|c|c|c|c|c|}
\hline \multirow{2}{*}{ Parameters } & \multicolumn{2}{|c|}{$M_{14}$} & \multicolumn{2}{c|}{$M_{41}$} \\
\cline { 2 - 5 } & Dysplasia & Adenocarcinoma & Dysplasia & Adenocarcinoma \\
\hline$Z_{1}$ & $0.19 \pm 0.023$ & $0.09 \pm 0.012$ & $0.055 \pm 0.01$ & $0.13 \pm 0.019$ \\
\hline$Z_{2}$ & $0.23 \pm 0.039$ & $0.12 \pm 0.017$ & $0.11 \pm 0.015$ & $0.21 \pm 0.036$ \\
\hline$Z_{3}$ & $0.73 \pm 0.11$ & $1.81 \pm 0.24$ & $2.87 \pm 0.43$ & $0.94 \pm 0.14$ \\
\hline$Z_{4}$ & $1.12 \pm 0.17$ & $2.18 \pm 0.31$ & $3.29 \pm 0.53$ & $1.62 \pm 0.27$ \\
\hline
\end{tabular}

The following quantitative criteria of differentiation of benign and malignant changes were determined due to the analyzed mechanisms of laser polarization autofluorescence:

- the Mueller-matrix image $M_{14}(m \times n)$ of a histological section of benign tumor is characterized by greater values of statistical moments of the $1^{\text {st }} Z_{1}\left(M_{14}\right)(2.1$ times $)$ and $2^{\text {nd }} Z_{2}\left(M_{14}\right)(2$ times $)$ orders. For statistical moments of higher orders the reverse tendency is typical $-Z_{3}\left(M_{14}\right)$ (2.5 times decrease) and $Z_{4}\left(M_{14}\right)(2.4$ times decrease).

- statistically third-order structures of polypeptide chains of collagen and myosin can be differentiated by the following properties: $Z_{1}\left(M_{41}\right)$ - increase by 2.4 times; $Z_{2}\left(M_{41}\right)$ - increase by 1.95 times; $Z_{3}\left(M_{41}\right)$ - decrease by 3 times and $Z_{4}\left(M_{41}\right)$ - decrease by 2 times.

Within both groups of histological sections by statistical approaches the sensitivity $\left(S e=\frac{d}{d+p} 100 \%\right)$ and specificity ( $S p=\frac{u}{u+h} 100 \%$ ) of the technique of Mueller-matrix mapping of laser polarization fluorescence of protein networks were determined, where $d$ and $p$ are the amount of right and wrong diagnoses within the group 1; $u$ and $h$ - the same within group 2.

For different Mueller-matrix rotation invariants of laser polarization autofluorescence the following results were obtained - Table 2.

Table 2. Sensitivity and specificity of Mueller-matrix technique of mapping of laser polarization fluorescence of the

\begin{tabular}{|c|c|c|}
\hline Parameters & $M_{14}$ & $M_{41}$ \\
\hline $\mathrm{Se}\left(Z_{i}\right), \%$ & 80 & 93 \\
\hline$S p\left(Z_{i}\right), \%$ & 75 & 85 \\
\hline
\end{tabular}

Thus, the statistical analysis of spectral-selective Mueller-matrix fluorescent invariants proved to be efficient in the task of differential diagnostics of benign and malignant changes of uterus wall. 


\section{CONCLUSIONS}

1. The model of laser polarization autofluorescence of biological tissues was suggested in which the mechanisms of optical anisotropic absorption, autofluorescence and birefringence were taken into consideration.

2. The interconnections between statistical parameters characterizing spectral-selective Mueller-matrix fluorescent images and the peculiarities of the mechanisms of porphyrin fluorescence in optically anisotropic histological sections of uterus wall biopsy were found.

3. The efficiency of the method of azimuthally invariant spectral-selective Mueller-matrix mapping of laser polarization autofluorescence of protein networks in the task of differentiation of benign and malignant tumors of uterus wall was demonstrated.

\section{REFERENCES}

[1]. Tower, T.T., Tranquillo, R.T., "Alignment Maps of Tissues: I. Microscopic Elliptical Polarimetry," Biophys. J. 81, p.p. 2954-2963 (2001).

[2]. Smith, M.H., Burke, P., Lompado, A., Tanner, E., Hillman, L.W., "Mueller matrix imaging polarimetry in dermatology," Proc. SPIE 3991, p.p. 210-216 (2000).

[3]. Shribak. M., Oldenbourg. R., "Techniques for Fast and Sensitive Measurements of Two-Dimensional Birefringence Distributions," Appl. Opt. 42, p.p. 3009-3017 (2003).

[4]. M.H Smith, "Interpreting Mueller matrix images of tissues," Proc. SPIE 4257, $82-89$ (2001).

[5]. Wang X., Wang, L.V. "Propagation of polarized light in birefringent turbid media: A Monte Carlo study," J. Biomed. Opt. 7, p.p. 279-290 (2002).

[6]. Angelsky, O.V., Bekshaev, A.Ya., Maksimyak, P.P., Maksimyak, A.P., Mokhun, I.I., Hanson, S.G., Zenkova, C.Yu., Tyurin, A.V., "Circular motion of particles suspended in a Gaussian beam with circular polarization validates the spin part of the internal energy flow," Optics Express 20, p.p. 11351-11356 (2012).

[7]. Bekshaev, A. Y., Angelsky, O. V., Hanson, S. G., Zenkova, C. Y., "Scattering of inhomogeneous circularly polarized optical field and mechanical manifestation of the internal energy flows," Phys. Rev. A 86, p.p. 023847 (2012).

[8]. Angelsky, O.V., Maksimyak, P.P., Perun, T.O. "Dimensionality in optical fields and signals," Applied Optics, 32, p.p. 6066-6071 (1993).

[9]. Angelsky, O. V., Ushenko, Y.A., "The Degree of Mutual Anisotropy of Biological Liquid Crystals Net during the Diagnostics of Human Tissues Birefringence," Advances in Optical Technologies 2010, p.p. 321275 (2010).

[10]. Angelsky, O.V., Bekshaev, A.Ya., Maksimyak, P.P., Maksimyak, A.P., Hanson, S.G., Zenkova, C.Yu., "Orbital rotation without orbital angular momentum: mechanical action of the spin part of the internal energy flow in light beams," Optics Express 20, p.p. 3563-3571 (2012).

[11]. Bard, M.P., Amelink, A., Skurichina, M., Noordhoek, V., Hegt, R., Duin, P., Sterenborg, H.J., . ,Hoogsteden, H.C, Aerts, J.G. , "Optical spectroscopy for the classification of malignant lesions of the bronchial tree," Chest. 129, p.p 995-1001 (2006).

[12]. Beamis J.F., Ernst, A., Mathur, P., Yung, R., Simoff, M., "A multi-center study comparing autofluorescence bronchoscopy to white light bronchoscopy," Lung Cancer 41, p.p. S49-S50 (2003).

[13]. Häussinger, K., Pichler, J., Stanzel, F., Markus, A., Stepp, H., Morresi-Hauff, A., Baumgartner, R., "Autofluorescence bronchoscopy: the D-light system," Interventional Bronchoscopy 30, p.p. 243-252 (2000).

[14]. Herth, F., Becker, H., "Autofluorescence bronchoscopy-a comparison of two systems (LIFE and D-Light)," Respiration 70, p.p. 395-398 (2003).

[15]. Hung, J., Lam, S.,. Leriche, J.C, Palcic, B., "Autofluorescence of normal and malignant bronchial tissue," Lasers Surg. Med. 11, p.p. 99-105 (1991).

[16]. Kamath, A.V., Chhajed, P.N., "Role of bronchoscopy in early diagnosis of lung cancer," J. Chest Dis. Allied Sci. 48, p.p. 265-269 (2006). 
[17]. Grossweiner, L.I., Blum, A., Goyal, G.C., "Advances in Experimental Medicine and Biology. Methods in Porphyrin Photosensitization," 193, p.p. 181-192 (1985).

[18]. Savenkov, S.N., Marienko, V.V., Oberemok, E.A., Sydoruk, O.I., "Generalized Matrix Equivalence Theorem for Polarization Theory," Phys. Rev. E. 74, p.p. 605-607 (2006).

[19]. Arteaga. O., Nichols. S., Kahr, B., "Mueller matrices in fluorescence scattering," Opt. Lett. 37, p.p. 28352837 (2012).

[20]. Alfano, R., Tata, D., Cordero, J., Tomashefsky, P., Longo, F., Alfano, M., "Laser induced fluorescence spectroscopy from native cancerous and normal tissue," IEEE Quantum Electron. 20, p.p. 1502 (1984).

[21]. Ushenko, A.G., Pishak, V.P., "Laser Polarimetry of Biological Tissue: Principles and Applications," Handbook of Coherent-Domain Optical Methods: Biomedical Diagnostics, Environmental and Material Science," 1, p.p. 93-138, (2004).

[22]. Angelsky, O.V., Ushenko, A.G., Ushenko, Yu.A., Pishak, V.P., Peresunko, A.P., "Statistical, Correlation and Topological Approaches in Diagnostics of the Structure and Physiological State of Birefringent Biological Tissues," Handbook of Photonics for Biomedical Science," p.p. 283-322 (2010).

[23]. Ushenko, Y.A., Boychuk, T.M., Bachynsky, V.T., Mincer, O.P., "Diagnostics of Structure and Physiological State of Birefringent Biological Tissues: Statistical, Correlation and Topological Approaches," Handbook of Coherent-Domain Optical Methods," p.p. 107-148 (2013).

[24]. Ushenko, Yu.A., Bodnar, G.B., Koval, G.D., "Classifying optical properties of surface-and bulk-scattering biological layers with polarization singular states," Journal of Innovative Optical Health Sciences 6, p.p. 1350018 (2013).

[25]. Ushenko, Yu.A., "Statistical structure of polarization-inhomogeneous images of biotissues with different morphological structures," Ukr. J. Phys. Opt. 6, p.p. 63-70 (2005).

[26]. Ushenko, Yu. A., Peresunko, A. P., Baku, B.A., "A New Method of Mueller-Matrix Diagnostics and Differentiation of Early Oncological Changes of the Skin Derma," Advances in Optical Technologies, p.p. 952423 (2010).

[27]. Ushenko, Yu.A., Telenga, O.I., Peresunko, A.P., Numan, O.K., "New parameter for describing and analyzing the optical-anisotropic properties of biological tissues," J. Innov. Opt. Health Sci. 4, p.p. 463-475 (2011).

[28]. Ushenko, Yu.A., "The feasibilities of using the statistical, fractal and singular processing of hominal blood plasma phase images during the diagnostics and differentiation of mammary gland pathological states," J. Innov. Opt. Health Sci. 5, p.p. 1150001 (2012).

[29]. Sroka, R., Baumgartner, R., Buser, A., Ell C., Jocham, D. , Unsoeld, E.,"Laser assisted detection of endogenous porphyrin in malignant diseases," Proc. SPIE 1641, p.p. 99-105 (1991).

[30]. D'Hallewin, M.A., Kamuhabwa, A.R., Roskams, T., De Witte, P.A., Baert, L., "Hypericin based fluorescence diagnosis of bladder carcinoma," BJU Int 89, p.p. 760-763 (2002).

[31]. D’Hallewin, M.A., Bezdetnaya, L., Guillemin, F., "Fluorescence detection of bladder cancer: a review," Eur. Urol. 42, p.p. 417-425 (2002). 